\title{
Versiones medievales inéditas de varios romances en un romancerillo manuscrito fragmentario
}

\section{Inedited Medieval Versions of Various Romances in a Fragmentary Manuscript 'Romancerillo'}

\author{
Mariano de la CAMPA \\ Universidad Autónoma de Madrid \\ mariano.campa@uam.es \\ BELINDA GARCÍA BARBA \\ Universidad Autónoma de Madrid \\ bgb1967@yahoo.es
}

La aparición de un testimonio desconocido con versiones medievales de romances trovadorescos supone una importante contribución a la historia del romancero antiguo. El manuscrito fragmentario de principios del siglo XVI contiene 5 romances cortesanos, uno de ellos desconocido en la tradición antigua, El Infante cautivo, que ha sobrevivido en la tradición oral moderna judeo española de Oriente.

Palabras Clave: romancero trovadoresco, poesía de cancionero, manuscrito, infante cautivo, siglo XVI

An important contribution to the history of romancero antiguo (old ballads) has been the discovery of an unknown text containing medieval versions of troubadour ballads (romances trovadorescos). The fragmentary manuscript, dating from early 16 th Century, contains 5 courtisan ballads, one of the unknown to the old tradition, El infante cautivo, which has survived in the Eastern Judeo-Spanish oral modern tradition.

KEYWORDS: romancero trovadoresco, poesía de cancionero, manuscript, infante cautivo, $16^{\text {th }}$ Century

ntes que los romances aparecieran impresos en forma de libro al me-
diar el siglo XVI, en el Cancionero sin año (1547-1549) y la Silva de
Zaragoza (1550), los impresores y mercaderes de libros ya habían 
descubierto el negocio editorial de la venta de romances en una forma más perecedera: los pliegos sueltos (Menéndez Pidal, Romancero, II, cap. XIII, \$ 5; Rodríguez-Moñino, Diccionario y Manual).

La documentación sobre el Romancero anterior o coetánea a los primeros pliegos impresos es muy escasa (MenéndezPidal, Romancero, II, cap. XIII, $\ 14,19)$. Se trata de romances o citas sueltas de versos incluidos en manuscritos, papeles sueltos o billetes. ${ }^{1}$

Los romances que se nos han conservado copiados en manuscritos, en cartapacios y volúmenes poéticos de fines del siglo xv y primeros años del XVI nos permiten hacer una valoración actual sobre el Romancero de entonces. Su testimonio nos hace ver la penetración del Romancero en círculos cortesanos más que ofrecernos una imagen del género en esa época. Los poetas cancioneriles, trovadores, componían para la sociedad cortesana poesías en forma de romances y glosas que se recogieron en los cancioneros de fines del siglo xv (Cancionero de Londres, Cancionero musical de Palacio, Cancionero general de 1511). ${ }^{2}$

En esta documentación manuscrita e impresa conservada se atestigua la presencia del Romancero en formas diversas. Por una parte encontramos los poemas compuestos en metro romance por los poetas trovadores; el tema amoroso es el leitmotiv en todas estas composiciones en las que los poetas se complacen en ofrecer la angustia amorosa teñida de desesperanza y exasperación ante la dureza o negativa de la amada: "Gritando va el caballero", "Maldita seas ventura”, "Si se está mi corazón”, "Contaros en que me vi” (Romeu, La música, 73-74; Catalán, "Romances trovadorescos”, Parte 1a, cap. XII). Por otra parte, los poetas cortesanos gustaban de recordar el Romancero viejo que había adquirido entre la buena sociedad la más alta consideración, convirtiendo al género Romancero en un género aristocrático. Estos romances viejos se readaptaban al nuevo estilo cancioneril unas veces en forma de glosas (El prisionero, Rosafresca, Fontefida, Durandarte, Conde Claros, glosados

\footnotetext{
${ }^{1}$ El texto más antiguo que conocemos es la versión de La dama y el pastor que copió un estudiante mallorquín, Jaume de Olesa, en un cuaderno manuscrito fechado en 1421. Tres romances coleccionados por Juan Rodríguez del Padrón en el Cancionero de Londres: Conde Niño, Rosaflorida y Montesinos, El caballero burlado (véase Morley, "Chronological”; Menéndez Pidal, Romancero, cap. XII, $\$ 4$, 5; Piacentini, Ensayo, Marín, Arcebispo y Marín y Pedrosa, "Un texto arcaico").

${ }^{2}$ Di Stefano ha subrayado que los textos publicados en el siglo xvi no pueden considerarse información equilibrada para conocer la temática del Romancero en su conjunto, pues el repertorio impreso responde a una selección impuesta por los gustos "oficiales" de la España de entonces (“La difusión”, 374).
} 
por G. Sánchez de Badajoz, N. Núñez, F. Pinar, F. de León), otras veces contrahaciendo los poemas (el de los Infantes de Lara, el de Roncesvalles, el de Lanzarote, etc.), y en otras ocasiones incorporándolos a la poesía cantada cortesana (Conde Claros, Lanzarote, Durandarte, La muerte de Alexandre). Ahora bien, el canto y la glosa condicionaban el texto romancístico acortándolo; la supresión de versos favorecía la agilidad del texto musicado o glosado, lo que confirió a los romances una gran popularidad (Menéndez Pidal, Romancero, cap. XII, \$ 11).

\section{Hallazgo de UN ROMANCILlo DE C. [1511-1515]}

Recientemente, al realizar tareas de catalogación y clasificación de documentos con contenido romancístico en el Archivo Menéndez Pidal (AMP) con objeto de completar las descripciones bibliográficas del Catálogo del Archivo Menéndez Pidal/ Goyri, ${ }^{3}$ recuperamos una breve nota que remitía de forma muy sucinta a la existencia de romances en un manuscrito de la Biblioteca Nacional de Madrid. ${ }^{4}$

El manuscrito 17.994 de la Biblioteca Nacional de Madrid es un manuscrito facticio formado por papeles de los siglos XVI, XVII y XVIII. La

\footnotetext{
${ }^{3}$ El Archivo Menéndez Pidal/Goyri del Romancero (AMP), iniciado y organizado por Ramón Menéndez Pidal y María Goyri, es el de mayor riqueza existente para el estudio del Romancero Pan-hispánico. Alrededor de 25.000 documentos forman los fondos de este importante depósito de la tradición romancística hispana. Organizado temáticamente, el Archivo Menéndez Pidal/Goyri no contaba con un índice o catálogo descriptivo detallado de todos sus fondos. Consiguientemente y con el propósito de crear un Archivo Informatizado del Romancero Pan-hispánico, la Fundación Ramón Menéndez Pidal viene llevando a cabo, desde 1990 (con la colaboración y apoyo de la Dirección General de Bellas Artes y Archivos, la Dirección General del Libro, Bibliotecas y Museos, la Fundación Ramón Areces, la Dirección General de Investigación Científica y Técnica y la Universidad Complutense de Madrid), la descripción pormenorizada e informatización de todos los documentos existentes en el Archivo mediante un "Proyecto de Catalogación, Informatización y Digitalización del Archivo Menéndez Pidal/Goyri”. El proyecto está siendo desarrollado por un equipo formado por Inés de la Cruz González-Cutre, Belinda García Barba, Javier Gómez Gómez, María González Piñeiro y Joaquín López Martínez, bajo la dirección de Diego Catalán. Fruto de esta labor es la publicación que se encuentra en prensa (Sirmio-Quaderns Crema) de un primer tomo del catálogo que recoge los datos de los documentos referidos a romances históricos, Catálogo analitico del Archivo romancístico Menéndez Pidal/Goyri, vols. I y II: Romances de tema nacional (e indices).

${ }^{4}$ En la tarea de documentación bibliográfica el equipo contó con la colaboración de Mariano de la Campa, bibliotecario de la Fundación Menéndez Pidal.
}

Medievalia 50, 2018, pp. 23-49 
procedencia dispar de los papeles se confirma por la existencia de varias numeraciones parciales que han sido unificadas por una moderna foliación a lápiz. El manuscrito, encuadernado en pergamino, perteneció a la biblioteca de don Pascual Gayangos y consta de 100 folios más una hoja de portada donde se puede leer: "Varias composiciones críticas relativas a los acontecimientos políticos de don Juan de Austria". ${ }^{5}$

Las composiciones que nos interesan aparecen en los folios 97-100, que formaban un cuadernillo independiente con su propia numeración, paginado del 3-13, la página 14 en blanco. Falta delante una hoja que correspondería a las páginas 1-2, y en medio faltan dos hojas que corresponderían a las páginas 7-8, 9-10. Se conservan las páginas 3-4, 5-6, 11-12, 13. De tal forma que actualmente en el manuscrito se corresponden la numeración moderna 26 y antigua del siguiente modo: f. $97 \mathrm{r}-97 \mathrm{v}$ (= pp. 3-4), f. $98 \mathrm{r}-98 \mathrm{v}$ (= pp. 5-6), f. 99r-99v (= pp. 11-12), f. 100r (= p. 13), f. 100v (= en blanco). El texto copiado a dos columnas.

Aunque en ningún momento aparecen nuestros textos fechados podemos deducir por el tipo de letra que fue copiado a fines del siglo XV o principios del siglo XVI. Las filigranas que aparecen en los ff. 97 y 98 representan una mano con una flor de seis pétalos (Briquet, Les filigranes, $n^{\circ} 11.165$ ) que a continuación reproducimos y que creemos corresponde a un papel salido de un molino de Perpignan de hacia 1505, lo que conviene bien al tipo de letra y al contenido del texto:

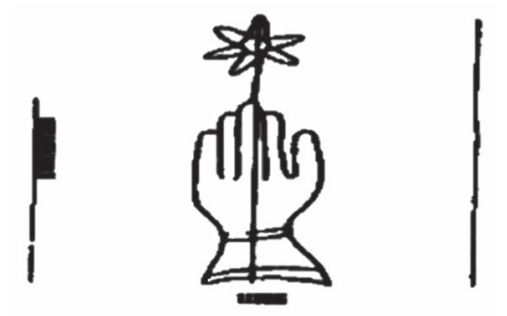

[Resulta verdaderamente complejo poder fechar no solo el manuscrito sino la la composición de los propios poemas. Si en un primer momento nos decantamos por la fecha de 105, guiados por la filigrana, ahora creemos

${ }^{5}$ El exlibris de la biblioteca de Pascual de Gayangos estampado en la cubierta y en el f. 1 . En el catálogo de la biblioteca de Gayangos aparece el manuscrito con el No 761 y se describe así: "Poesías varias, casi todas satíricas, relativas principalmente a los acontecimientos políticos con D. Juan de Austria y a los del siglo XVIII, L. de la ép.; 92 h. fol. Perg.. Véase Roca, Catálogo. 
que es más acertado, siguiendo las observaciones de Beltrán que relacionan el romancerillo fragmentario con el Cancionero general de 1511, fecharlo entre 1511 y 1515 o poco después, en los primeros años del reinado del Emperador.]

\section{DESCRIPCIÓN DEL CONTENIDO}

DEL MANUSCRITO FRAGMENTARIO

1) f $97 \mathrm{ra}(=$ p. 3). Rromançe. "Rrosa fresca, rrosa fresca..."

2) f. 97rb-98va (= pp. 3-6). La glosa deste rromançe es de Pinar. "Quando yos quise querida...”

3) f. 98va (= p. 6). Rromance. "Por Dyos te rruego carcelero..."

4) f. 98va-98vb (= p. 6). La glosa deste rromance es de Mexya. "La memoria se nos cuenta..." (Se glosan 4 hemistiquios por la pérdida de los folios siguientes)

5) f. 99ra-99rb (= p. 11) (La glosa de Fonte Frida cortada por pérdida de los dos folios anteriores. Se glosan 12 hemistiquios de los 26 que tiene el romance) "...Pues quyen tal pérdida pierde..."

6) f. $99 \mathrm{rb}(=$ p. 11). Rromance, "Contaros en que me vy..."

7) f. 99va-99vb. La glosa es de Vyuero. "Sy desdychas consolasen..."

8) f. 99vb-100ra (= pp. 12-13). Rromance. "Maldyta seas ventura..."

9) f. 100ra-b (= p, 13). La glosa es de Núñez, "Partydo de my beuyr..." (Se glosan 8 hemistiquios de los 12 de que consta el romance, pues dejó de copiarse el texto, f. 100v en blanco).

Por lo tanto, el cuadernillo de 4 folios que estuvo formado de 13 páginas contenía hasta donde podemos reconstruir:

A) Romances trovadorescos:

1) Rosa fresca. ${ }^{6}$

\footnotetext{
${ }^{6}$ Rosa Fresca: Cancionero General de Hernando del Castillo, 1511, f. 132 (y en las eds. siguientes de 1514, 1517, 1520, 1527, 1535, 1540, 1557 y 1573); Segunda parte del Cancionero General, 1552, f. 40v; Cancionero de romances, s. a., f. 230v. (y también en el de Miles, f. 245v.; 1550 , f. 244v, y en sus sucesivas eds. de 1555, 1568 y 1581); Primera Silva, 1550, f. 153; Dechado de galanes (como noticia, pues no se conserva. Colón lo describe); en los pliegos sueltos: DicARM no 918, 658, 668, 771 a 774, 1035, 1038, 1039; y en los manuscritos siguientes: Cancionero del British Museum, f. 12v. y 29 (glosa de Pinar); Cancionero de Pedro del Pozo, f. 25; Madrid, Biblioteca Nacional, Ms. 5593, f. 42. También aparece citado por Gil Vicente en su Comedia Thebayda y en multitud de ensaladas de romances del siglo xvi (La vida del
} 
2) El infante cautivo.

3) Fonte Frida. ${ }^{7}$

4) Contaros en que me vi. ${ }^{8}$

5) Maldita seas ventura.

B) Glosas de romances:

1) La glosa de Pinar a Rosa fresca.

2) La glosa de Mexía a El infante cautivo.

3) La glosa de Fonte Frida.

4) La glosa de Vivero a Contaros en que me vi.

5) La glosa de Núñez a Maldita seas ventura.

[Las relaciones entre este romancerillo fragmentario y el Cancionero General de 1511 han sido puestas de manifiesto por Vicenç Beltrán en un excelente trabajo que estudia el romancero viejo y el romance trovadoresco desde el reinado de los Reyes Católicos hasta el de la Reina Juana (1474-1516). Para Beltrán se trata no solo de un romancerillo de poesía cortés sino de un repertorio de glosas que viene a completar los escasos testimonios con los que contamos para esta época (Beltran, El Romancero, cap. 2).]

estudiante pobre, Glosa peregrina, Disparates de Sarabia, etc.). Conocemos también su música gracias a Salinas, De musica libri septem, 1577, 411.

${ }^{7}$ Fonte Frida: Cancionero General de Hernando del Castillo, 1511, f. 133 (y en las eds. siguientes de 1514, 1517, 1520, 1527, 1535, 1540, 1557 y 1573); Segunda parte del Cancionero General, 1552, f. 44; Cancionero de romances, s. a., f. 230v. (y también en el de Miles, f. 246; 1550, f. 245, y en sus sucesivas eds. de 1555, 1568 y 1581); Fernández de Constantina, Cancionero, f. 58; Primera Silva, 1550, f. 153v.; Libro de cincuenta romances (Morbecq, fragm.); en los pliegos sueltos; DicARM no 654, 870, 936, 1038, 1039, 1170. También figura en el Cancionero del British Museum, f. 19 y 102 (glosa de Tapia); Cancionero de Palacio, f. 82v.; Cancionero de Pedro del Pozo, f. 25v.; Madrid, Biblioteca Nacional, Ms. 3724, f. 158v.

${ }^{8}$ Contaros en que me vi: Cancionero General de Hernando del Castillo, 1511, f. 133 (y en las eds. siguientes de 1514, 1517, 1520, 1527, 1535, 1540, 1557 y 1573).

${ }^{9}$ Maldita seas ventura: Cancionero General de Hernando del Castillo, 1511, f. 133v. (y en las eds. siguientes de 1514, 1517, 1520, 1527, 1535, 1540, 1557 y 1573); Cancionero de romances, s. a., f. $248 \mathrm{v}$. (y también en el de 1550, f. $262 \mathrm{v}$., y en sus sucesivas eds. de 1555, 1568 y 1581); Primera Silva, 1550, f. 173v.; Primera Silva, Barcelona, 1550, f. 145 (y también en la ed. de Barcelona, 1552, f. 135); en los pliegos sueltos: DicARM no 654; y en el Cancionero del British Museum, f. 26v. y el Cancionero de Pedro del Pozo, f. 25v. 


\section{TRANSCRIPCIÓN DEL MANUSCRITO}

Pág. 3 (fol. mod. 97)

$$
\begin{aligned}
& \text { rromançe } \\
& \text { rosa f[r] } \text { esca rrosa fresca } \\
& \text { tan garryda y con amor } \\
& \text { quando yostuuen mys braços } \\
& \text { no vos supe seruyr non } \\
& \text { y agora [cos] seruyrya } \\
& \text { non vos pu[edo] [yo] aver no } \\
& \text { y agora cos seruyrya } \\
& \text { non vos puedo yo aver no } \\
& \text { v[uest]ra fue la culpa amygo } \\
& \text { v[uestr]a fue que mya no } \\
& \text { enbyastes me vna carta } \\
& \text { con vn v[uest]ro seruydor } \\
& \text { y en lugar de rrecabar } \\
& \text { el dyxera otra rrazon } \\
& \text { que erades casado amygo } \\
& \text { alla en tyerras de leon } \\
& \text { y tenes muger hermosa } \\
& \text { y hyjos como vna flor } \\
& \text { quyen os lo dyxo señora } \\
& \text { no vos dyxo verdad no } \\
& \text { que yo nunca entren castylla } \\
& \text { ny alla en tyerras de leon } \\
& \text { syno quando era pequeño } \\
& \text { que no sabya de amor } \\
& \text { la glosa deste } \\
& \text { rromançe es de } \\
& \text { Pynar. }
\end{aligned}
$$

quando yos quyse queryda

sy supyera conoceros

nostuuyera yo perdyda

ny acucyara yo la vyda

agora para quereros

$y$ por ques byen que padezca 
desta causa my dolor

llamos yo syn que os merezca

rrosa fresca rrosa fresca

tan garryda y con amor

llamos yo con boz planyda

llena de gran conpasyon

con ell alma entrystecyda

del angustya doloryda

que a çufrydo el coraçon

quel se haze myl pedaços

yo muero do quyer que vo

pues que por mys enbaraços

quando yostuuen mys braços

non vos supe servyr no

Pág. 4 (fol. mod. 97v)

no por que os ovyese errado

con pensamyento de errar

mas sy me days por culpado

pues publyco my pecado

deveysme de perdonar

no por que quandos seruya

my querer os deseruyo

mas por que paso solya

$y$ agora que os seruyrya

no vos puedo yo aver no

rrespuesta de la

.dama.

sy supyerades amores

como supystes engaños

fueron $v$ [uest $]$ ros mys fabores

yo byuyera syn dolores

y escusaranse dos daños

del v[uest]ro vos soys testygo

del myo callo le yo

pues pyerdo quando lo dygo 
$\mathrm{v}$ [uest $]$ ra fue la culpa amygo

$\mathrm{v}$ [uest $]$ ra fue que mya no

$v$ [uest $]$ ra fue la culpa dello

myo el dolor de sentyllo

v[uest]ro el plazer de hazello

myo el pesar de sabello

yagora doble en [dezi]llo

dygolo con pe[na harta]

syendo vos el causador

syn que todo se departa

enbyastesme vna carta

con vn v[uest] ro seruydor

no me acuerdan las rrasones

quel mensajero de enojos

deshyzo las conclusyones

y despynte los rrynglones

en ell agua de los ojos

no cuydandose acordar

quera v[uest]ra my afycyon

el comyença de hablar

y en lugar de rrecabar

el dyxera otra rrazon

Pág. 5 (fol. mod. 98)

dyxo lo que sospechaua

y lo que de vos creya

por que quando el me hablaua

con el seso autoryzaua

todo quanto me dezya

dyxo que os dyese castygo

pues que me dystes pasyon

dyxo lo que no desdygo

que erades casado amygo

alla en tyerras de leon

yo le pregunte rrogando

que el me dyxese verdad

Medievalia 50, 2018, pp. 23-49 
sy me lo dezya burlando

el me rrespondyo jurando

que era mas en cantydad

y sy os fuy desdeñosa

y os trate con dysfauor

nos marauylles de cosa

pues tenes muger hermosa

y hyjos como vna flor

dyzele el caua

llero.

byen dyre yo desamado que por malos boluedores pues masydo levantado vn testymonyo falsado con dychos engañadores que os hazen mal secutora $y$ pena quyen no peco por que tal dycho a desora quyen os 10 dyxo señora no vos dyxo verdad no

por que el dyxo con malycya lo que no hyz ny pense mas sy de tal ay [ynd]ycya hagase de my justycya que yo lo consentyre y por mayor marauylla yo muera syn confesyon syn rreparo y syn mansylla sy yo nunca entren castylla ny alla en tyerras de leon

Pág. 6 (fol. mod. 98v)

no son mas.

y por este juramento $\tan$ fuerte que veys que hago 

quyero luego en vn momento declarar my pensamyento por salyr deste enpalago con tal fe que yos enpeño my fe de buen amador que no entre ny yo lo sueño syno quando era pequeño que no sabya de amor rromance.

por dyos te rruego carcelero quanto te puedo rrogar que mafloxes las cadenas y me quytes el collar carcelero con manzylla luego me las fue afloxar echeme sobr[e] vn almena por my cuerpo asolazar y vy cancas de castylla que juntan con portugal

la glosa

deste rromance es de Mexya.

la memorya se nos cuenta ser mal de los apartadas por que aquesta rrepresenta al deseo catormenta todos los byenes pasados y pues es tan lastymero el dolor del desear por dyos te rruego carcelero quanto te puedo rrogar por quen verme yo apartado no de pena desygual preso y suelto my cuydado con dolor atormentado 


$$
\begin{aligned}
& \text { duele la vyda my mal } \\
& \text { y en apretarme las penas } \\
& \text { te rruega my sospyrar } \\
& \text { que mafloxes las cadenas } \\
& \text { y me quytes el collar }
\end{aligned}
$$

Pág. 11 (fol. mod. 99r)

pues quyen tal perdyda pyerde

verse byua es mas peor

que ny poso en rrama verde

ny en prado que tenga flor

sy la muerte me llevara

nostuuyera qual esto

que sy ell agua hallo clara

turuya la beuya yo

tengo el coraçon partydo

desventura le partyo

que no quyero aver marydo

por que hyjos no aya yo

mas quyero penar syn ellos

mas quyero my perdycyon

que no aver plazer con ellos

ny menos consolacyon

y pues note pydo abrygo

ny amygo tengo amor

dexame tryste enemygo

malo falso mal traydor

.no son mas.

no me des ya mas fatiga

que harta me tengo yo

que no quyero ser tu amyga

ny casar contygo no 
rromance

contaros en que me vy quando era enamorado yo malas noches avyendo peores dyas pasando por servycyo de my amyga sy la vyese de my vando

Pág. 12 (fol. mod. 99v)

la glosa es de

Byuero

sy desdychas consolasen quanto consuelo ternya el syn ventura de my sy dysfauores amasen tan amado yo serya quan tryste syenpre me vy desastrado desastrado y desamado pues perdy contaros en que me vy quando era enamorado

de mal andança conplyda syenpre me vy tan conplydo quan menguado de plazer $y$ a vos tan poca seruyda quan ganada yo perdydo con rrazon devyera ser yo penando yo penando y byen amando yo muryendo yo malas noches avyendo peores dyas pasando no ay mas 
quando pense que tenya

halgo vencyda la pena

halleme ser mas penado

quando mas glorya tendya

halle la pena mas llena

halleme a my desamado

esperando

esperando y conportando

gran fatyga

por servycyo de my amyga

sy la vyese de my vando

rroman

ce

maldyta seas ventura

casy me hases andar

desterrado de mys tyerras

de donde soy natural

por amar a vna señora

la qual no devyera amar

adamela por my byen

y salyome por my mal

por que ame donde nospero

galardones alcançar

Pág. 139

(fol. mod. 100)

por hazer plazer amor

amor me hyzo pesar

la glosa es de

Nuñez

partydo de my bevyr

do agora muero byuyendo

saque de byuo el sentyr

por acabar de moryr

y ando contyno muryendo

camynando con trystura 


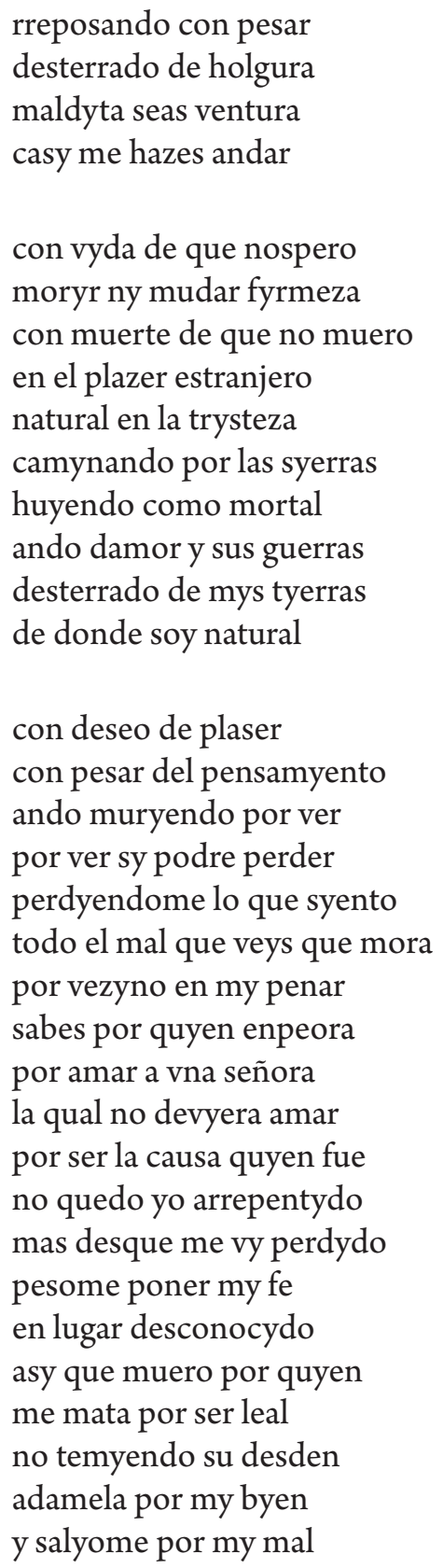




\section{romathas}

38

\section{$\uparrow$} mofafefor ropof fector tall gatziod y collatuoz quatioo yof turuct itpo bzacos no boo fiupe fertyz tho ya croza marsiyzya thoth 200 ing no ya gosa cos lesteveya nombospueso yoabez tio via fic la culpa anygo bia flic que nua tio etibyaftes ine buacasta cori vit bio fezuyaz venlugaz arvecabaz dopoerd otza 12430ti

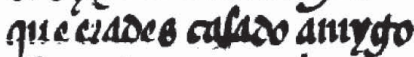
alla cintperas a loon y tence untog Geztitofa y gylos atmo bua floz quien os toaye perioza no bos tyoo beradono que yo uuna entrencaftella tiy alla city cruse aleon fyno quation c2 a pequeño que nofabya or amor

\author{
Laglofacefte \\ rtotmanctics \\ - pynat. \\ 1 quatrooyofouplequequa \\ iv (uppless conceres \\ Hoftumpesa yopezoyed \\ in acuquas yola byea \\ agorapazd quarezos \\ y poz ques byen queparga \\ aelta curfa ine ocloz \\ llamos yo fun qucogmezga \\ i mola pelar. viofafesta \\ tati jartyoa y wituatuoz
} 1

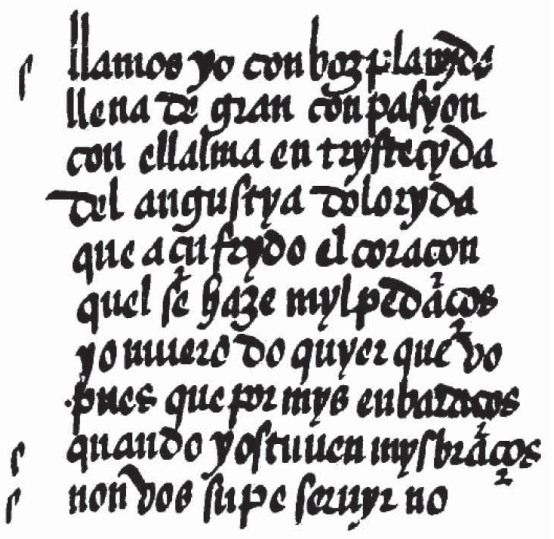


f no pre qucos obvele cratas conpcrifaniy cil to tecraz thas fymeans forculpaso pules publy ar my pe atoo to beve me oe pezanaz no póz que quatrog Rz!na miqueses os of furvo mas pos que pabololya f pa gora que oe fezuyza $i$ thobos pucos yo dicatio rrefpueftada - Dalma.

\section{1}

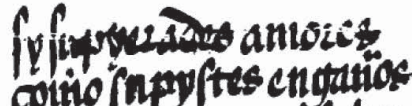
ficroti uzes mine fabozes yo bynycza fontolozes. ycluparancoap oanos

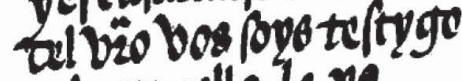
cel inyo callo le ye

1 viaf fick la culpa amygo

1. bia fue que trya no biafucla aulpa ocllo nive cl oolor oc centyllo bio cl plazer Di hazello inyo cl pesazo fitilla yigura noble ant joenoo boe el ralidacoz fyil quic todo fercpazta

chlyaftes inc bua rata

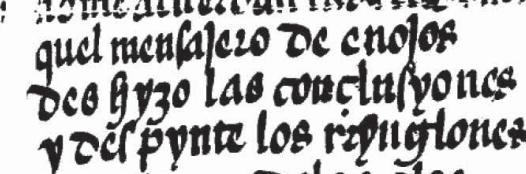 y achynte los riphiglos ne cuipaniode acoious

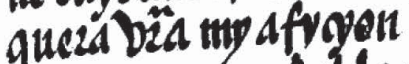 ilcomyena ac gablaz 1' jenlugazoc trecribaz al oyera otza razon 


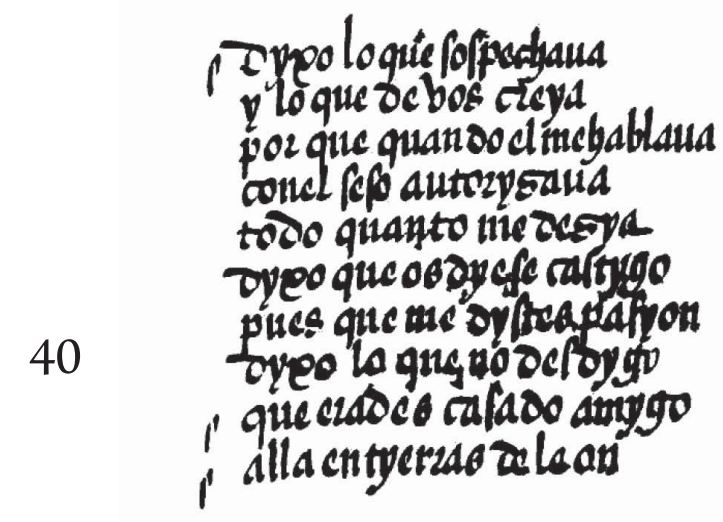


. Hofon thas.

1 yporc]te] uramento tati fice te quibsyequegayo quyczo livego on onmomionto Dedazar wn penfamy crito $202 \mathrm{Cly}_{2}$ torte enpalag, contal fe que pos en petio

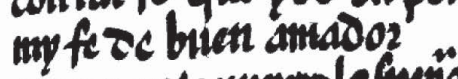
que no entre'ny jolofwina, truo quatio e un pequetio i que no falyd or amoz

- rovinana.

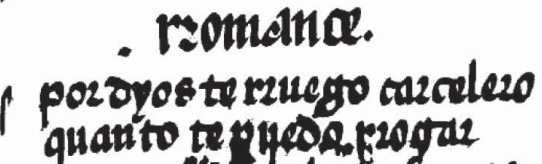
que inafr. de lad robanas vine quy catcesor hansylla litego tere IS fic a flobat coteme falor silal nicua

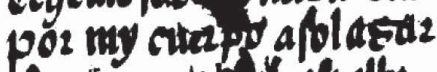

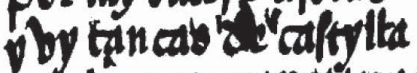
atic intran contortitgal Laglofo

\section{Dofterromana es $\mathfrak{d}$ - merys.}

Lancmozya penos cucuta pez mal Tolos apartadodo por que aquefurreprefenta alocfeo ra tozmenta tooos log bycpes palatoos4 ypues of tain laftruteso doolor oclocfear

$\uparrow$ 1 por oyes te rucgo carcelezo
quatito te pucoe reggar

1 poz quen beeneyo afutado ho oc pena ze Prgual prefo y fucl to mparyoado con ooloz ator merititos cuele labjoa my mal penapectarme Laspenas

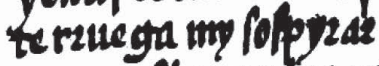
que ma floper las aroenas 1 yine quy tes el collaz 
I' fuce quipen talpecoyod yeso bez c byula es mas peoz

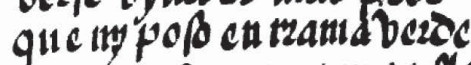
nyen fizaso que tengafloz

1' fylamiterte inc llouaza noftu upera qual efte

1 que /y cllaguaballo daza turuya la benya yo

tingo cl coraconpartyos zes bentura 'leparty? que no quyere abez maztoo por que Gylos npayayo

mat quy cro pernaz fyncllos 111as quyczo my paoygon que ne aber plaser conellos in menos confolagon. $\int$ ypucanote proo abzygo ny anygo tritos amoi oceane tzyite cucinygo malo falfoul trapdo

. nofon mas.

i nome Degyamus futygr quic yasta true tetigo'y. que no quyczo (crttlatiggen ny adfa autygo no

\section{romatux}

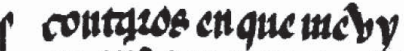
quattao czactiantozo yomalas no tyes abyenso pecess cyas pafano poz leztivopo de iny atugga pla byefe re nutuano 


\section{Laghlof sed} byilero.

Pfy ocfingtase confolafen quante winfuclo tezriya el fynbenttuadomy

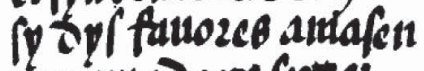
tan attazoyos festas quan tzy fre fyectipzemeby ic $\operatorname{sectsin}$

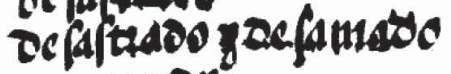
preamezoy

1 19 cou talos an gac mely quanos cza chandmozaro

os nal atwo anda conpliton

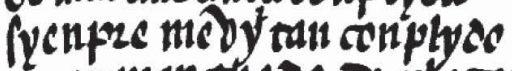
gut an mengliados as plasez y abos tain pora sez y yad quangando yo pezs yos con reasan ze hyeza per yo penando yopenambo y byen amando yo thutrycisos yo inalas nodges abyeño peczes ayas padaino

\section{Modym.1s}

1 quanas prufe que te nya dalco biengea lapecia Galteme Yeimas pendio quando mas glozya tentiya gadle la pena inde Hoina fillle the atuy arfatiaro iffecanas clperando y conptortatios gzan fatyga.

por fertuv aro ac iny artugta filla byefe oc my balles

rroms

I maloyta (cas Wechtiusa cafy me gaedo andodz auferiaca mps tyerzas oc ourose foy nattizat poz a amat abna fáfora laqual ine ac byecquatma ioamela por my byen y falyoutc for iny inal

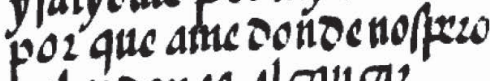
gallazosince alrail az: 


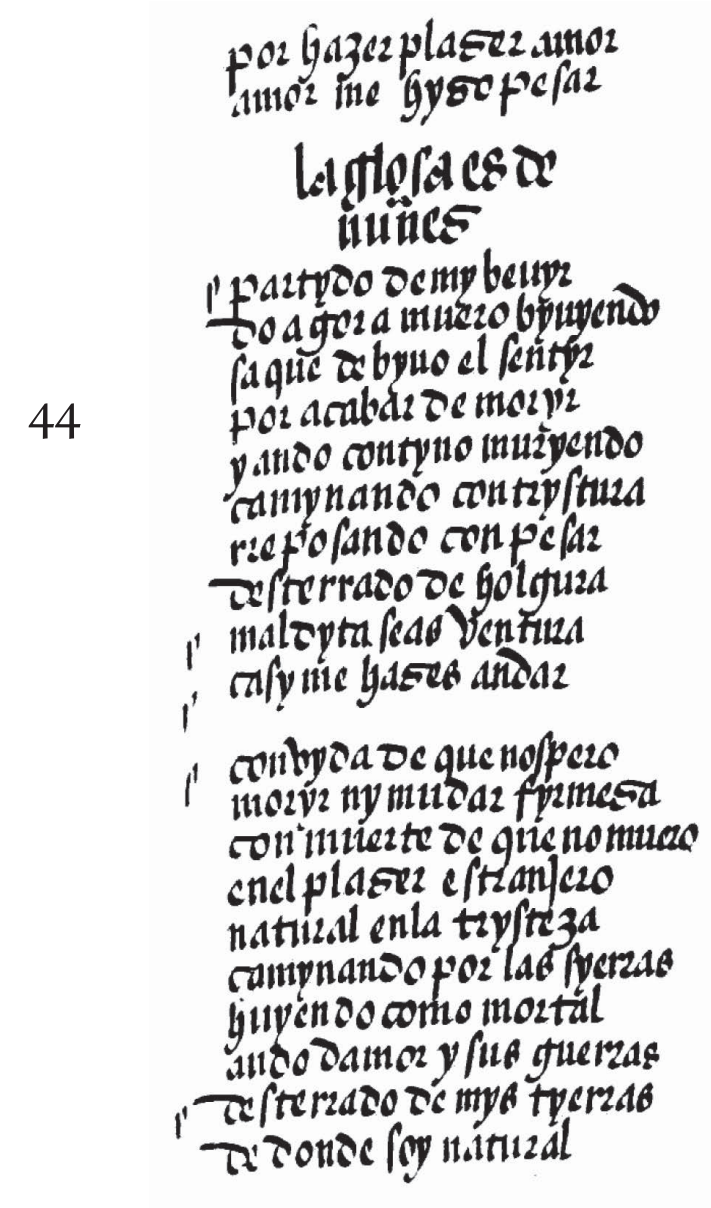

i colloefeo scplarez conpe fas ad pen fantento atiog milzyenoo por ve? por bes ypdou peroce peravenaome loquefonto tore cl tral quebsequemoza pet ve gyne cu thy pent pabel por quyen cupesia 1 Yoz amul dina fenoza laqual noocbyadmaz

$p$ por ce la caufa quyen fue tho quicos yo ariepentroo mate ac que me by pragas pelonc ponc my fe

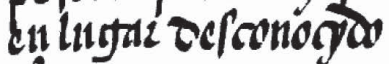
a) quis inte 20 porquyen me inata-poz fezleal ne temydroo fitaclocn 1' doancla por my byen 1 
El texto más curioso de todos es el de El infante cautivo, pues revela la antigua existencia de un romance que hasta ahora solo nos era conocido en la tradición judeo española de Oriente.

La versión medieval ahora descubierta cuenta únicamente:

- ¡Por Dios te ruego, carcelero, cuanto te puedo rogar, que maflojes las cadenas y me quites el collar! Carcelero, con mancilla, luego me las fue aflojar. Echéme sobre un almena por mi cuerpo asolazar, y vi cancas ${ }^{10}$ de Castilla que juntan con Portugal.

Son varias las versiones de la tradición sefardí moderna que conocemos, unas publicadas, otras inéditas. Las más antiguas fueron copiadas en el siglo XVIII; otras recogidas por los colectores de la tradición oral moderna. En el Archivo Menéndez Pidal/Goyri se conservan 13 versiones y una cita suelta del romance en un manuscrito. ${ }^{11}$ Otras 9 versiones hemos localizado en diversas publicaciones. ${ }^{12}$ Son todas muy similares entre sí.

Ofrecemos como muestra una versión inédita de Sarajevo, copiada en los Mss. hebraicos I y II, de la segunda mitad del siglo XVIII, transcrita por Manuel Manrique de Lara en 1911, y conservada en el Archivo Menéndez Pidal/Goyri:

— iQue me quitex las cadenas y me afloxes el colán

2 que ondas me dan de muerte que me quería matar!-

\footnotetext{
${ }^{10}$ No hemos podido documentar la palabra cancas. La lectura es segura. Desafortunadamente la glosa del romance se corta tras ser glosados los 4 primeros hemistiquios y no podemos saber si la palabra glosada es la que aparece en el romance autónomo.

${ }^{11}$ Descritas por Armistead (El Romancero judeo-español), 8 de Sarajevo, 2 de Esmirna, 1 de Rodas, 1 de Salónica, 1 de Salónica-Sofía, de las que se han publicado tres; dos en Armistead (El Romancero judeo-español [u op. cit.]) y una en Benmayor (Romances judeo-españoles). La nota manuscrita en caracteres hebreos se halla en un ejemplar de los poemas de Nağara (Venecia, $5359=1600$ ), citado por Armistead (El Romancero judeo-español [u op.cit.]).

${ }^{12}$ Molho (Literatura sefardita) publicó una versión, otras tres Levy (Chants), dos más Attias ("Ceror"), otra en Armistead, Hassán, Silverman ("Un nuevo testimonio") reproducida en Armistead y Silverman (En torno), y dos en Benmayor (Romances judeo-españoles).
}

Medievalia 50, 2018, pp. 23-49 
Carcelero por piedad cadenas le fue a quitar.

4 Pensó que era por su bien, salió que era por su mal.

Metiólo en altas torres, más altas de la ciudad.

6 Siete puertas tiene la torre, todas siete en un andar; las tres daban para Francia, y las tres para Portugal

8 y la más chiquita de ellas daba encima de la mar.

Alzó sus ojos a lexos cuanto más los pudo alzar, 10 vido venir nueva galena navegando por la mar.

Las cuerdas tiene de ibrixim de aquel ibrixin gatanlí

12 las velas tiene de seda y de aquel rico cendal

el casco tiene de un bel cristal.

14 ¡Más vale fortuna en tierra que no bonanza en mar!

$2 \mathrm{~b}$ colán: cinturón. $12 \mathrm{a}$ ibrixim: cordón de seda, en turco.

12b gatanlí: del sitio donde el cordón fue elaborado.

[La profesora Avviva Garribba que se ha ocupado de estudiar el texto antiguo del Infante cautivo y las versiones sefarditas de oriente (Garriba, "Il romance”, 94-100) procedentes de la tradición oral moderna considera que existen dudas para considerarlo en su origen como un texto trovadoresco, sin embargo, sigue explicando, nada puede afirmarse con seguridad hasta no tener más datos sobre la historia que cuenta el romance (Garriba, "Algo más”, 153).]

El profesor Samuel Armistead se ha interesado por este romance y en sus trabajos hace referencia a su origen enigmático; cree que puede tener alguna relación con otros romances sefardíes conservados en las comunidades de Marruecos: El cautiverio de don Francisco y el Conde Arnaldos (Armistead, Hassán, Silverman, "Un nuevo testimonio", 210-212; Armistead, El Romancero judeo-español, 297-300; Benmayor, Romances judeo-españoles, 106-109 y Armistead y Silverman, En torno, 73-75). Ciertamente, en las versiones que se conservan en el Archivo Menéndez Pidal/Goyri de El cautiverio del príncipe Francisco (una versión de Tánger: $T$, y dos de Alcazarquivir: $A_{1}, A_{2}$ ) se conserva la fórmula en la que el carcelero alivia al prisionero: "-Carcelero, carcelero / así (si $\left.\mathrm{A}_{1}, \mathrm{~A}_{2}\right)$ Dios te guarde de (del $\left.\mathrm{A}_{1}, \mathrm{~A}_{2}\right)$ male, // súbeme (subidme $\mathrm{A}_{2}$ ) un poquito arriba, / me (que me $\mathrm{A}_{2}$ ) dará (de $\left.\mathrm{A}_{2}\right)$ un poquito el aire, // gozaré del viento fresco, / de las $\left(\right.$ la $\left.\mathrm{A}_{1}\right)$ tierras (tierra $\left.\mathrm{A}_{1}\right)$ de mi padre. - // El (omite $A_{1}, A_{2}$ ) carcelero por mancilla / le subió un poquito al aire //".

Aunque no pretendemos realizar en este momento un estudio comparativo minucioso del texto medieval, con las copias del siglo XVIII y las versiones orales modernas, nos parece que, en su origen, el texto nada tuvo que 
ver con el Conde Arnaldos, pues ni se asoma al mar, ni ve aproximarse una nave maravillosa, ni el navegante que va en ella entona una canción, tal como sucede en las versiones modernas. Sí coincide, en cambio, con el Cautiverio del príncipe Francisco en suplicar al carcelero que le suelte.

Muchos problemas, por el momento no fáciles de resolver, presenta aún este texto, digno de un estudio más amplio. Parece tratarse de un texto del Romancero viejo incorporado a la poesía glosada de Cancionero. Si es así, el romance viejo podría haber sido acortado narrativamente y ser las versiones modernas donde mejor se conserva la totalidad del tema. Pero también cabe que el texto del romance fuera tal como se nos ha conservado en el manuscrito de la Biblioteca Nacional, y entonces podría pensarse que las versiones sefardíes están contaminadas por otro romance.

\section{HALlAZGO DE UNA HOJA SUELTA CON VERSOS ROMANCÍSTICOS}

Otro manuscrito importante para el Romancero no tenido en cuenta es el manuscrito 7.896 de la Biblioteca Nacional de Madrid, de mediados del siglo XVI, con 622 folios. Recoge distintos escritos de Alvar Gómez de Castro en gran parte autógrafos. En el f. 356 se copian cinco pareados dieciseisílabos pertenecientes, quizá, a tres temas distintos, que no hemos podido identificar: ${ }^{13}$

Ya caualgaua Arnaldos
en vn cauallo ligero
la silla era de oro
y el freno de Toledo.
Ya caualgaua Arnaldos
en vn ligero cauallo
la silla era de oro
y el freno Toledano.
E[n] calças está el co[n]de
y en un jubó $[n]$ de co[n]traye
el pan tenía e[n] la mano los canes q[u]ería çeuare.

${ }^{13}$ Es un folio que nada tiene que ver con el manuscrito en que se encuentra, más bien parece un ensayo de escritura donde alguien escribió estos versos. 
Si mis colores graçiosas yo señor las he $\mathrm{p}$ [er] dido, mi brial rico de seda todo me le auíe ro[n]pido. Si v[uest] ro brial de seda todo os le auíe rasgado ricos somos mi s[eñor]a haga otro más loçano.

\section{BibLIOGRAFÍA}

Armistead, Samuel G., El Romancero judeo-español en el Archivo Menéndez Pidal. (Catálogo índice de romances y canciones), 3 vols., Madrid: Cátedra Seminario Menéndez Pidal, 1978.

Armistead, Samuel G., Iacob. M. Hassán y Joseph H. Silverman, “Un nuevo testimonio del romancero sefardí en el siglo XVIII”, Estudios Sefardíes, 1, 1978, 210-212.

Armistead, Samuel G. y Joseph H. Silverman, En torno al romancero Sefardí (hispanismo y balcanismo de la tradición judeo-española), Madrid: Seminario Menéndez Pidal, 1982.

AtтiAs, Moshe, “'Çeror rômansonth be-kth' y sel Sarayevo”, Shevet va-'Am, 2:7, 1973, 295-370.

[Beltrán, Vicenç, El Romancero: De la oralidad al canon, Kassel: Edition Reichenberger, 2016.]

Benmayor, Rina, Romances judeo-españoles de Oriente. Nueva recolección, Madrid: Cátedra Seminario Menéndez Pidal-Gredos, 1979.

Briquet, Charles M., Les filigranes. Dictionnaire historique des marques du papier. III. L-O, Hildesheim-Zürich-New York: Georg Olms Verlag, 1984 [1 $1^{\text {a }}$ ed. Leipzig, 1923].

CATAlán, Diego, "Romances trovadorescos incorporados al romancero tradicional moderno”, en Arte poética del Romancero oral. Parte $1^{a}$ Los textos abiertos de creación colectiva, Madrid: Siglo XXI, 1977, 291-324.

Di Stefano, Giuseppe, "La difusión impresa del Romancero antiguo en el siglo XVI”, Revista de Dialectología y Tradiciones Populares, 33, 1977, 373-411.

[Garribba, Aviva, Garribba, Aviva, "Il romance dell'Infante cautivo a Sarajevo", Estudios de Literatura Oral, 4, 1998, 85-101.]

[Garribba, Aviva, "Algo más sobre la única versión antigua del romance del Infante cautivo", en Rafael Beltrán (ed.), Historia, reescritura y pervivencia del 
Romancero. Estudios en memoria de Amelia García-Valdecasas, Valencia: Publicacions de la Universitat de València, 2000, 37-155.]

LeVY, IsAAC, Chants judéo-espagnols, t. III, Jerusalem; Edición del autor, 1971.

Marín Padilla, EnCarnación, "Arcebispo de Çaragoça". Romance castellano manuscrito del año 1429, Zaragoza: [1997].

Marín, Encarnación y José Manuel Pedrosa, "Un texto arcaico recuperado para la historia del romancero: una versión aragonesa manuscrita (1448) de Las quejas de Alfonso V", Madrid, Edición de los autores, 1999 [luego en Revista de Literatura Medieval, 12, 2000, 177-191].

Menéndez Pidal, Ramón, Romancero hispánico (hispano-portugués, americano y sefardí). Teoría e historia, 2 vols., Madrid: Espasa-Calpe, 1968 [1 1 ed. 1953].

Molho, Michael, Literatura sefardita de Oriente, Madrid-Barcelona: CSIC, 1960.

Morley, S. Griswold, "Chronological List of early Spanish Ballads", Hispanic Review, 13, 1945, 273-287.

Piacentini, Giuliana, Ensayo de una bibliografía analítica del romancero antiguo. Los textos (siglos XV y XVI). III. Los Manuscritos, Pisa: Giardini, 1994.

Roca, Pedro, Catálogo de los manuscritos que pertenecieron a D. Pascual de Gayangos existentes hoy en la Biblioteca Nacional, Madrid: s/e, 1904.

Rodríguez-Moñino, Antonio, Diccionario bibliográfico de pliegos sueltos poéticos (siglo XVI), Madrid: Castalia, 1970.

Rodríguez-Moñino, Antonio, Manual bibliográfico de Cancioneros y Romanceros, 4 vols., Madrid: Castalia, 1973-1978.

Romeu Figueras, José, La música en la corte de los Reyes Católicos. IV-1. Cancionero musical de Palacio (siglos XV y XVI). Volumen 3-A, Barcelona: CSIC, 1965. 